\title{
Electronic properties of grain boundaries in $\mathrm{Cu}(\mathrm{In}, \mathrm{Ga}) \mathrm{Se}_{2}$ thin films for different Ga-contents
}

\author{
Robert Baier ${ }^{\mathrm{a}, *}$, Jascha Lehmann ${ }^{\mathrm{a}}$, Sebastian Lehmann ${ }^{\mathrm{a}, \mathrm{b}}$, Thorsten Rissom ${ }^{\mathrm{a}}$, Christian Alexander \\ Kaufmann $^{\mathrm{a}}$, Alex Schwarzmann ${ }^{c}$, Yossi Rosenwaks ${ }^{c}$, Martha Ch. Lux-Steiner ${ }^{\mathrm{a}}$, Sascha Sadewasser ${ }^{\mathrm{a}}$ \\ ${ }^{a}$ Helmholtz-Zentrum Berlin für Materialien und Energie, Hahn-Meitner-Platz 1, 14109 Berlin, Germany \\ ${ }^{b}$ Solid State Physics, Lund University, Box 118, S-22100 Lund, Sweden \\ ${ }^{c}$ Department of Physical Electronics, School of Electrical Engineering, Tel Aviv University, Tel-Aviv 69978, Israel
}

\begin{abstract}
We present a study on the electronic properties of grain boundaries (GBs) in polycrystalline $\mathrm{Cu}(\mathrm{In}, \mathrm{Ga}) \mathrm{Se}_{2}$ (CIGSe) thin films. As grown as well as KCN-treated films were comparatively investigated. No influence of the chemical treatment on the electronic properties of GBs was found. GBs in general exhibited a large variation of their electronic properties. By means of a novel method of data analysis, both potential barriers for holes and electrons were found at GBs, in a range from $-118 \mathrm{mV}$ to $+114 \mathrm{mV}$, as well as uncharged GBs. No dependence of the electronic GB-properties on the Ga-content was found. Consequently we therefore conclude that there is no correlation between electronic GB-properties and the obtained maximum efficiencies of CIGSe thin film solar cells as a function of the Ga-content.
\end{abstract}

Keywords: CIGSe, grain boundaries, KPFM

\section{Introduction}

Thin-film solar cells based on the chalcopyrite material $\mathrm{Cu}(\mathrm{In}, \mathrm{Ga}) \mathrm{Se}_{2}$ (CIGSe) achieve the highest power conversion efficiency of all thin film photovoltaic devices[1]. Also they outperform their monocrystalline counterpart by far, yielding record efficiencies above 20\%[2]. This is unique among all solar cell materials. In general monocrystalline devices yield higher efficiencies, due to a higher crystalline quality and e.g. the absence of grain boundaries (GBs). GBs are commonly regarded as detrimental for solar cell performance, as they exhibit a high density of both structural and electronic defects and can act as recombination centers $[3,4]$. The record performance of thin film CIGSe solar cells is therefore, at least partly, attributed to a special behavior of the GBs in CIGSe. Despite a high research activity around this phenomenon, the physics underlying the electronic activity of GBs in CIGSe is still controversially discussed[5-10].

In principle three different models for the beneficial behavior of GBs in CIGSe exist: The "atomic relaxation model", the "structural barrier model", and the "electronic barrier model". The "atomic relaxation

\footnotetext{
* Corresponding author

Email address: robert.baier@helmholtz-berlin.de (Robert Baier)
} 
model" predicts that GBs in CIGSe do not create deep level defects within the band gap[7, 10]. Due to a large atomic relaxation in GB regions the defect levels are shifted into the valence band. As a result, GBs are electrically benign and not harmful to solar cell performance. The "structural barrier model", on the other hand, assigns a valence band offset to the CIGSe near-GB region, resulting from a $\mathrm{Cu}$ depletion[5, 11]. This downward shift of the valence band maximum acts as a potential barrier for holes, which reduces recombination at GBs. No interface charges are involved in this model. The third model, the "electronic barrier model", is based on the existence of positively charged defects around GBs $[12,13]$. Those defects lead to a downward band bending at GBs, the so called "local built-in potential". It also results in a negative potential barrier that reflects holes from GBs. Thereby recombination of electrons and holes is reduced along GBs and pathways for minority-carriers (electrons) to the front contact are created.

Based on the "electronic barrier model", Jiang et al.[14] concluded that the local built-in potential of GBs plays a significant role for device performance. They studied the band bending at GBs for CIGSe samples with varying $\mathrm{Ga} /(\mathrm{Ga}+\mathrm{In})$ ratio by KPFM. Up to $28 \%$ Ga-content they found a potential barrier of $\approx 150 \mathrm{mV}$. This barrier sharply droped to $0 \mathrm{mV}$ between $28-38 \%$. For a Ga-content higher than $38 \%$ they did not report on any local built-in potential. This characteristics of the local built-in potential agrees well with device performance. Device performance also sharply drops for a Ga-content above $\approx 45 \%$, while in theory a Gacontent of $65 \%$ provides the optimal band-gap for energy conversion. Because of this concurrence between device performance and local built-in potential at GBs Jiang et al. concluded on a direct correlation between these two observations.

Another study on the influence of $\mathrm{Ga}$ on the electronic properties of GBs was conducted by Romero et al.[15]. Applying electro-assisted scanning tunneling microscopy, they investigated the electron transport across CIGSe GBs for different Ga/(Ga+In) ratios. For a $\mathrm{Ga} /(\mathrm{Ga}+\mathrm{In})$ ratio of 1 they report on a significant barrier for electron transport across grain boundaries, whereas they did not detect any transport-barrier for a $\mathrm{Ga} /(\mathrm{Ga}+\mathrm{In})$ ratio of 0 .

In this article we revisit the influence of the $\mathrm{Ga} /(\mathrm{Ga}+\mathrm{In})$ ratio on CIGSe GBs again. We present an elaborated KPFM study on the influence of Ga-content on the electronic properties of GBs in polycrystalline CIGSe thin films. As grown as well as chemically treated CIGSe thin films were measured in several UHV KPFM-systems leading to new interesting insights in the physics of CIGSe GBs.

\section{Experimental}

Five CIGSe thin films, with $\mathrm{Ga} /(\mathrm{Ga}+\mathrm{In})$ ratio varying from 0 to 1 , were grown on Mo-coated float glass substrates by similar multi-stage co-evaporation processes inside the same evaporation chamber[16]. This growth process leads to Cu-poor composition of the samples. Reference solar cells were processed by chemical bath deposition of a CdS buffer-layer and subsequent sputter deposition of an i-ZnO/ZnO:Al double window layer. A Ni/Al grid was used as front contact. Table 1 provides an overview of the thin film- 


\begin{tabular}{l|l|l|l|l|l|l|l} 
Sample & $\frac{\mathbf{G a}}{\mathbf{G a}+\mathbf{I n}}$ & $\frac{\mathbf{C u}}{\mathbf{G a}+\mathbf{I n}}$ & $\begin{array}{l}\text { Thickness } \\
(\mu \mathbf{m})\end{array}$ & $\mathbf{V}_{O C}(\mathbf{m V})$ & $\mathbf{J}_{S C}\left(\mathbf{m A} / \mathbf{c m}^{2}\right)$ & $\begin{array}{l}\text { Fill-factor } \\
\mathbf{( \% )}\end{array}$ & $\begin{array}{l}\text { Efficiency } \\
(\mathbf{\%})\end{array}$ \\
\hline CIGSe-0 & 0.00 & 0.82 & 2.05 & $490.1 \pm 3.6$ & $36.9 \pm 0.7$ & $72.5 \pm 1.9$ & $13.1 \pm 0.4$ \\
CIGSe-33 & 0.33 & 0.86 & 1.72 & $633.1 \pm 4.2$ & $34.3 \pm 0.5$ & $69.9 \pm 0.5$ & $15.2 \pm 0.3$ \\
CIGSe-45 & 0.45 & 0.82 & 2.11 & $673 \pm 158$ & $28.5 \pm 0.8$ & $74 \pm 20$ & $14.1 \pm 4.9$ \\
CIGSe-76 & 0.76 & 0.80 & 2.00 & $718 \pm 134$ & $13.1 \pm 0.6$ & $51 \pm 6$ & $4.8 \pm 1.0$ \\
CIGSe-100 & 1.00 & 0.88 & 1.89 & $727 \pm 18$ & $13.4 \pm 0.6$ & $55 \pm 3$ & $5.3 \pm 0.4$
\end{tabular}

Table 1: Overview of thin film- and device-parameters of the different CIGSe thin films studied.

and device-parameters of all samples. Two different kinds of surface conditions were investigated in the course of this study: As grown and chemically treated samples. As grown samples didn't receive any surface treatment. They were exposed to ambient air prior to the measurements. Chemically treated samples were etched in $0.15 \mathrm{M}$ aqueous $\mathrm{KCN}$-solution for $2 \mathrm{~min}$ in order to remove surface oxides and further contamination. To prevent the KCN-treated samples from re-oxidation, they were kept under deionized water environment during the transfer to ultra high vacuum (UHV) conditions (base pressure $<10^{-10} \mathrm{mbar}$ ). Directly after introduction to UHV, all samples (as grown as well as chemically treated) were annealed at temperatures of $\approx 130^{\circ} \mathrm{C}$ for $30 \mathrm{~min}$ to remove residual water from the samples.

The effect of the chemical treatment was investigated in a XPS study on a $\mathrm{CuIn}_{0.71} \mathrm{Ga}_{0.29} \mathrm{Se}_{2}$ thin film similar to those in Table 1[17].

KPFM measurements at the Helmholtz-Zentrum Berlin (HZB) were performed in UHV using the amplitude modulation technique at the second resonance frequency of the cantilever for detection of the contact potential difference (CPD)[18]. The applied ac-voltage was $100 \mathrm{mV}$. For topography measurements the conventional frequency modulation technique at the first resonance frequency $\left(f_{0} \approx 75 \mathrm{KHz}\right)$ was utilized. All measurements were performed with Pt-Ir-coated Si cantilevers at a tip-sample distance $d \approx 10 \mathrm{~nm}$. Absolute work function $(\Phi)$ values were obtained from the CPD images by calibration measurements on highly ordered pyrolytic graphite. KPFM measurements at Tel Aviv University (TAU) were performed in UHV using the amplitude modulation technique at the second resonance frequency of the cantilever for detection of the contact potential difference (CPD). The applied ac-voltage was $50 \mathrm{mV}-150 \mathrm{mV}$. For topography measurements the conventional frequency modulation technique at the first resonance frequency $\left(f_{0} \approx 75 \mathrm{KHz}\right)$ was utilized. All measurements were performed with Pt-Ir-coated Si cantilevers.

\section{Data Analysis}

In general, it is not straight forward to identify GBs from KPFM measurements. Many studies in the past (including our own) consulted both work function- and topography images in order to identify GBs. This is clearly not the best approach, as it prefers electronically active GBs. An optimal way to identify GBs is electron backscatter diffraction (EBSD). Complementary EBSD/KPFM measurement would therefore be desirable, as they allow precise identification of GBs in KPFM. Unfortunately, those measurements have not 
been possible so far. Therefore, we here use the following approach to identify GBs:

Only topography images were consulted to determine the location of GBs. Thereby a misguiding by the work function images, which could lead to non-consideration of electronically inactive GBs, was prevented. The electronic behavior of GBs was extracted from work function images by taking line profiles, always perpendicular to the GB. Ten neighboring line profiles were averaged for the analysis of one GB, in order to minimize the noise level and the influence of work function variations along the GB. 20 GBs from 3 macroscopically different positions on the sample were analyzed for each Ga-content and surface treatment. This reduces the influence of possible inhomogeneities of the samples.

For the as grown series, KPFM measurements were carried out at both HZB and TAU, to exclude systematic errors. Measurements at HZB and TAU were conducted on samples from the identical batch and with a similar pre-treatment of the samples. Ten additional GBs from measurements at TAU were analyzed for each $\mathrm{Ga} /(\mathrm{Ga}+\mathrm{In})$ ratio.

In addition, the size of the CIGSe grains was extracted from KPFM topography images. 20 grains from macroscopically different sample positions were analyzed for each Ga-content. Here the grain shape was approximated as elliptic. The grain size was defined as the average of the grains' minor and major semiaxis.

Work function distributions were obtained by fitting the histogram of a work function image with Gaussian distributions[19]. The maximum of the fitted function is regarded as the images' work function. The FWHM of the main Gaussian function is used as a measure for the variation of the work function within an image.

\section{Results}

To gain information about the influence of oxidation on the CIGSe thin films a XPS-study was carried out at the beginning. It will be reported in detail elsewhere[17]. Here only a few essential facts are given. It was observed that CIGSe is relatively prone to oxidation. However, the oxidation behavior was found to be very different for the individual elements. As can be seen in Figure 1(a) In and Ga very quickly form surface oxides. After $100 \mathrm{~h}$ exposure to ambient air $25 \%$ of In, respectively $38 \%$ of Ga, on the surface was oxidized. In contrast, $\mathrm{Cu}$ and Se oxidize comparably slow. Respective oxides were only detectable after 1000h exposure to air, then yielding an oxide percentage of $14 \%$ and $33 \%$, respectivly. Figure 1(a) also shows the capability of the KCN-treatment to remove oxides from the surface. After the etching-procedure nearly all oxides are removed and the surface is left in a chemically clean state. Figure 1(b) illustrates the effect of oxidation and KCN-treatment on the average work function of CIGSe surfaces as determined by KPFM. Two samples, both from the same piece used for the XPS-study, were exposed to ambient air for 1h/500h, respectively. Due to oxidation, the work function of the sample exposed for $500 \mathrm{~h}$ is significantly lower, compared to the sample exposed for 1h. After the KCN-treatment, the work functions of both samples increase to similar values, due to the removal of oxides. Based on these results we treated all samples of the Ga/(Ga+In)-series 
with the same KCN-treatment, in order to remove surface oxides. In the following we present the results, comparatively to as grown samples.

Figure 2 shows $7 \mu \mathrm{m} \times 7 \mu \mathrm{m}$ images of the CIGSe- 0 thin film as grown and chemically treated. No distinct difference in topography is visible before and after the treatment. The average work function after the chemical treatment is increased by $\approx 600 \mathrm{meV}$ compared to the as grown thin film, due to removal of oxides. This value is in good agreement with the results in Figure 1(b) as well as with literature[19].

KPFM images of all CIGSe films of Table 1 are shown in Figure 3. The KPFM measurements clearly reveal variations of the films' grain size, depending on the Ga-content. The dependence, extracted from these and many other images, is illustrated in Figure 4. For 0\% Ga-content an average grain size of about $1.3 \mu \mathrm{m}$ is found. With increasing Ga-content the grain size decreases to $\approx 600 \mathrm{~nm}$ for a Ga-content of $100 \%$. The results are in good agreement with EBSD- measurements by Abou-Ras et al.[20]. Figure 4 also displays the work function distribution of the KPFM images from Figure 3. The work function of all films is generally found to be similar, varying between $\approx 4.7 \mathrm{eV}-5.0 \mathrm{eV}$. However, we note that an interpretation of this behavior is very difficult, since explicit additional information about the position of the Fermi-level inside the band gap would be required. This information is very hard to obtain, especially at the surface where Fermi-level pinning, due to a high defect density, is likely.

The analysis of the electronic behavior at GBs in dependence of the $\mathrm{Ga} /(\mathrm{Ga}+\mathrm{In})$ ratio yielded interesting results. Figure 5(a) shows the work function behavior for the series of as grown thin films. For $0 \%$ Ga-content almost all electronically active GBs display a negative potential barrier of up to -64mV. For $\mathrm{Ga} /(\mathrm{Ga}+\mathrm{In})$ ratios from 0.33 to 1 , both positive and negative potential barriers were observed, ranging from $-118 \mathrm{mV}$ to $+100 \mathrm{mV}$. However, no trend of the electronic behavior can be found with increasing Ga-content. Additionally, about $25 \%$ of all GBs do not show any electronic activity, independent of the Ga-content. Figure 5(b) illustrates the results for the chemically treated thin films. In principle, the results are similar to the as grown ones. Again, a large scattering of the electronic behavior of the GBs is observed. For all Gacontents, both positive and negative potential barriers are detected, in a range from $-83 \mathrm{mV}$ to $+114 \mathrm{mV}$. No correlation between the $\mathrm{Ga} /(\mathrm{Ga}+\mathrm{In})$ ratio and the electronic properties of GBs can be assigned. The number of electronically inactive GBs increased, compared to the as grown films. For CIGSe-33 and CIGSe-76 more than $50 \%$ of the GBs were determined to be electronically inactive.

\section{Discussion}

The applied KCN-treatment has proven to be an effective method to remove contaminations and oxides from CIGSe thin films[17]. Furthermore it creates a defined work function state on the surface independent of the previous oxidation level, as can be seen in Figure 1(b). Considering this issue, plus the fact that the treatment is a quick and uncomplicated procedure, KCN-etching exhibits to be a very suitable method 
for surface preparation of CIGSe thin films. It provides a clean, defined, and reproducible surface state, regardless of the state of previous oxidation.

Within this study the electronic properties of GBs in CIGSe thin films were investigated with KPFM. In KPFM, in contrast to Atomic Force Microscopy, the electrostatic force between tip and sample is nullified dynamically, which yields information about the local work function distribution of a sample. Due to the long range nature of the electrostatic force, the work function change at a nanoscale surface potential variation, i.e. a $\mathrm{GB}$, is subject to an averaging effect. The magnitude of the averaging effect strongly depends on the tip-sample distance. A tip-sample distance of $d \approx 10 \mathrm{~nm}$, for example, which was used for all experiments reported here, yields only $\approx 25 \%$ of the full work function change[21]. Consequently, all potential barriers reported in this article are probably underestimated by up to a factor of $\approx 4$. Nevertheless, to avoid any confusion, we will report here only on the actually measured work function change. More detailed information about this will be reported elsewhere[22].

In general, KPFM measurements did not yield big differences of the electronic GB-properties between as grown and chemically treated thin films. Both positive and negative potential barriers of up to $\approx 100 \mathrm{mV}$, as well as uncharged GBs, were observed for almost all CIGSe films studied. This points out that KPFM is in fact sensitive to electronic properties of the GB interface and not only detects surface effects. Otherwise one would expect a distinct difference between as grown and chemically treated electronic GB-properties, as the electronic information from GBs, as obtained after the chemical treatment, should be buried by contamination for the as grown thin films.

The potential barriers given in Figure 5 do not suggest any correlation between the work function behavior at GBs and the Ga-content. This is in contradiction to a previous study[14], which reports a direct correlation between local built-in potential and Ga-content, respectively device performance. A possible explanation for the different result might be the different experimental set-up. While all results reported here were obtained under UHV conditions, experiments of Ref.[14] were performed in ambient air, where the tip-sample distance is typically much larger than under UHV conditions. This leads to a decrease in KPFM sensitivity [21, 2325], which gets more important with smaller grain size (see Figure 4). Another reason could be the samples itself. It is well known that the growth process has a strong influence on the electronic properties of CIGSe thin films. Hence, different electronic properties of the samples used in Ref. [14] compared to these used in our study are also conceivable, even if the growth processes in both studies were similar.

The analysis of CIGSe GBs generally revealed a large variation of the electronic behavior. We attribute these variations to different structural properties, namely to different orientations of GBs. It is well-established in literature that the symmetry of GBs influences their electronic properties. For example, $\sum 3$ GBs were found to be electronically inactive[26], whereas $\sum 9$ GBs revealed a negative potential barrier of $\approx 90 \mathrm{mV}$ in earlier KPFM studies[9]. Since grains grow randomly in polycrystalline material, all kinds of GB-orientations are expected to appear in those films. For the analysis of GBs shown in Figure 5 all 
different kinds of GB-orientations were considered. Hence, the large scattering of the electronic properties does not surprise, but it rather was to be expected. However, for an exact understanding of the connection between GB-orientation and electronic activity, an orientation-dependent study would be desirable.

The numerous uncharged GBs and positive potential barriers, as reported in Figure 5, are an unusual finding at CIGSe GBs using KPFM. Earlier studies mostly revealed negative potential barriers near GBs. The reason for this finding probably also originates from the method of GB analysis (see Section 3). The identification of GBs only by topography images avoided a preferential selection of GBs with negative potential barriers. As a result, we obtain an analysis of the electronic properties of GBs, which also includes uncharged GBs and GBs with positive potential barriers.

A consequence of this new analysis is that non of the three common GB-models introduced in Section 1 is in agreement with the results. The "atomic relaxation model" predicts that defects do not create deep levels in GB regions, due to a large atomic relaxation. This should correspond to defect-free conductionand valence-bands, and therefore, by KPFM we would not expect to detect any potential barriers.

The also purely theory-based "structural barrier model" assigns a valence band offset to near-GB regions, which results from a Cu-depletion[5]. While a valence band offset is found for both, $0 \%$ and $100 \%$ Gacontent, for 100\% Ga-content an additional conduction band offset is reported from calculations[11]. This conduction band offset should (assuming similar electron affinities for GBs and grain interior) lead to a negative potential barrier at GBs for films with $100 \%$ Ga-content, while Ga-free films should not show any potential barriers at GBs in KPFM. However, our KPFM results show similar barriers for all Ga-contents, which is not in agreement with expectations for the "structural barrier model".

The third model, the "electronic barrier model", is based on the existence of charged defects around GBs. While positively charged donor-like defects lead to a downward band bending at GBs, and hence to negative potential barriers, negatively charged acceptor-like defects result in an upward band bending at GBs, and hence result in positive potential barriers. Therefore, this model could explain our experimental results. However, it is unclear how the remarkable energy conversion efficiencies of CIGSe thin film solar cells can be explained. While negative potential barriers result in a barrier for holes at GBs, thereby reducing recombination at GBs, positive potential barriers are not expected to have a similarly beneficial effect for photovoltaic applications. GBs with positive potential barrier would reflect electrons back towards the grain, where holes are majority-carriers, and therefore recombination is likely.

Consequently, our results can not be fully explained by any of the GB models available to explain the remarkable energy conversion efficiencies of CIGSe thin film solar cells. However, it might not even be possible to explain the high record efficiencies considering only one model. It is possible that a combination of different effects accounts for the results observed in Figure 5. For example, the appearance of uncharged GBs might be explained by the "atomic relaxation model". Additionally, charged defects (e.g. due to antisite occupation, Na-diffusion) at some GBs could lead to potential barriers. 
It also has to be considered, that GBs' bulk properties might be different to those at the surface, as observed by KPFM. Nevertheless, even in this case the surface GB properties are still relevant for solar cell performance, because the junction to the n-type window layer will be formed right at this interface. This being said, a chemical modification of the electronic GB-properties (in the surface near region) in the course of solar cell production in fact also seems conceivable[27, 28].

\section{Conclusions}

We have presented a study on the electronic properties of GBs in CIGSe thin films. As grown as well as chemically treated films were studied by means of UHV-KPFM. The chemical treatment turned out to be very suitable for surface preparation, as it removes surface oxides. However, the treatment did not effect the electronic properties of GBs. GBs showed, in general, a large scattering of their electronic properties. Negative as well as positive potential barriers, ranging from $-118 \mathrm{mV}$ to $+114 \mathrm{mV}$, were found, as well as uncharged GBs. No dependence of the electronic GB-properties on the Ga/(Ga+In)-Ratio of the CIGSe-films could be detected. Consequently, the study does not suggest any correlation between electronic GB-properties and performance deficits of CIGSe thin film solar cells with high Ga-content and hence higher band gap.

\section{Acknowledgments}

The authors gratefully acknowledge financial support from the Bundesministerium für Umwelt, Naturschutz und Reaktorsicherheit (BMU) under contract \# 0327559H.

[1] M. A. Green, K. Emery, Y. Hishikawa, W. Warta, Solar cell efficiency tables (version 37), Progress in Photovoltaics: Research and Applications 19 (1) (2011) 84-92.

URL http://dx.doi.org/10.1002/pip.1088

[2] P. Jackson, D. Hariskos, E. Lotter, S. Paetel, R. Wuerz, R. Menner, W. Wischmann, M. Powalla, New world record efficiency for $\mathrm{Cu}(\mathrm{In}, \mathrm{Ga}) \mathrm{Se} 2$ thin-film solar cells beyond 20\%, Prog. Photovolt: Res. Appl. (Paper presented at 25th EU PVSEC WCPEC-5, Valencia,Spain,2010). URL http://dx.doi.org/10.1002/pip.1078

[3] H. Leamy, G. Pike, C. Seager (Eds.), Grain Boundaries in Semiconductors, North-Holland, New York, 1982.

[4] A. P. Sutton, R. W. Balluffi, Interfaces in Crystalline Materials, Oxford University Press, USA, 1995.

[5] C. Persson, A. Zunger, Anomalous grain boundary physics in polycrystalline CuInSe2: The existence of a hole barrier, Phys. Rev. Lett. 91 (26) (2003) 266401.

[6] D. Marron, S. Sadewasser, A. Meeder, T. Glatzel, M. C. Lux-Steiner, Electrical activity at grain boundaries of $\mathrm{Cu}(\mathrm{In}, \mathrm{Ga}) \mathrm{Se} 2$ thin films, Phys. Rev. B 71 (3) (2005) 033306.

[7] Y. Yan, C. S. Jiang, R. Noufi, S.-H. Wei, H. R. Moutinho, M. M. Al-Jassim, Electrically benign behavior of grain boundaries in polycrystalline CuInSe2 films, Phys. Rev. Lett. 99 (23) (2007) 235504.

[8] U. Rau, K. Taretto, S. Siebentritt, Grain boundaries in Cu(In, Ga)(Se, S)2 thin-film solar cells, Appl. Phys. A 96 (1, Sp. Iss. SI) (2009) 221.

[9] M. Hafemeister, S. Siebentritt, J. Albert, M. C. Lux-Steiner, S. Sadewasser, Large neutral barrier at grain boundaries in chalcopyrite thin films, Phys. Rev. Lett. 104 (19) (2010) 196602.

[10] H. Moenig, Y. Smith, R. Caballero, C. A. Kaufmann, I. Lauermann, M. C. Lux-Steiner, S. Sadewasser, Direct evidence for a reduced density of deep level defects at grain boundaries of $\mathrm{Cu}(\mathrm{In}, \mathrm{Ga}) \mathrm{Se}-2$ thin films, Phys. Rev. Lett. 105 (11) (2010) 116802 .

[11] C. Persson, A. Zunger, Compositionally induced valence-band offset at the grain boundary of polycrystalline chalcopyrites creates a hole barrier, Appl. Phys. Lett. 87 (21) (2005) 211904.

[12] J. Seto, Electrical properties of polycrystalline silicon thin films, J. Appl. Phys. 46 (12) (1975) 5247.

[13] S. Schuler, S. Nishiwaki, J. Beckmann, N. Rega, S. Brehme, S. Siebentritt, M. C. Lux-Steiner, Charge carrier transport in polycrystalline CuGaSe2 thin films, in: Proceedings of the 29th IEEE conference, IEEE,Piscataway, 2002 , p. 504. 
[14] C.-S. Jiang, R. Noufi, K. Ramanathan, J. AbuShama, H. Moutinho, M. Al-Jassim, Does the local built-in potential on grain boundaries of $\mathrm{Cu}(\mathrm{In}, \mathrm{Ga}) \mathrm{Se} 2$ thin films benefit photovoltaic performance of the device?, Appl. Phys. Lett. 85 (113) (2004) 2625.

[15] M. Romero, C. Jiang, R. Noufi, M. Al-Jassim, Lateral electron transport in Cu(In,Ga)Se2 investigated by electro-assisted scanning tunneling microscopy, Appl. Phys. Lett. 87 (17) (2005) 172106.

[16] C. A. Kaufmann, R. Caballero, T. Unold, R. Hesse, R. Klenk, S. Schorr, M. Nichterwitz, H. W. Schock, Depth profiling of $\mathrm{Cu}(\mathrm{In}, \mathrm{Ga}) \mathrm{Se} 2$ thin films grown at low temperatures, Solar Energy Mater. Solar Cells 93 (6-7) (2009) 859, 17th International Photovoltaic Science and Engineering Conference, Fukuoka, JAPAN, DEC 03-07, 2007.

[17] J. Lehmann, S. Lehmann, I. Lauermann, T. Rissom, C. Kaufmann, M. C. Lux-Steiner, S. Sadewasser, in preparation.

[18] C. Sommerhalter, T. Matthes, T. Glatzel, A. Jäger-Waldau, M. C. Lux-Steiner, High-sensitivity quantitative Kelvin probe microscopy by noncontact ultra-high-vacuum atomic force microscopy, Appl. Phys. Lett. 75 (2) (1999) 286.

[19] S. Sadewasser, Surface potential of chalcopyrite films measured by KPFM, Phys. Status Solidi A 203 (11) (2006) 2571.

[20] D. Abou-Ras, R. Caballero, C. A. Kaufmann, M. Nichterwitz, K. Sakurai, S. Schorr, T. Unold, H. W. Schock, Impact of the Ga concentration on the microstructure of CuIn1-xGaxSe2, Phys. Stat. Sol. (RRL) 2 (3) (2008) 135.

[21] C. Leendertz, F. Streicher, M. C. Lux-Steiner, S. Sadewasser, Evaluation of Kelvin probe force microscopy for imaging grain boundaries in chalcopyrite thin films, Appl. Phys. Lett. 89 (11) (2006) 113120.

[22] R. Baier, C. Leendertz, M. C. Lux-Steiner, S. Sadewasser, in preparation.

[23] T. Hochwitz, A. K. Henning, C. Levey, C. Daghlian, J. Slinkman, Capacitive effects on quantitative dopant profiling with scanned electrostatic force microscopes, The 3rd International Workshop on the Measurement and Characterization of Ultra-Shallow Doping Profiles in Semiconductors 14 (1) (1996) 457-462.

URL http://link.aip.org/link/?JVB/14/457/1

[24] U. Zerweck, C. Loppacher, T. Otto, S. Grafström, L. Eng, Accuracy and resolution limits of Kelvin probe force microscopy, Phys. Rev. B 71 (12) (2005) 125424.

[25] S. Sadewasser, C. Leendertz, F. Streicher, M. C. Lux-Steiner, The influence of surface topography on Kelvin probe force microscopy, Nanotechnology 20 (50) (2009) 505503.

[26] S. Siebentritt, S. Sadewasser, M. Wimmer, C. Leendertz, T. Eisenbarth, M. C. Lux-Steiner, Evidence for a neutral grain-boundary barrier in chalcopyrites, Phys. Rev. Lett. 97 (14) (2006) 146601.

[27] T. Glatzel, M. Rusu, S. Sadewasser, M. C. Lux-Steiner, Surface photovoltage analysis of thin CdS layers on polycrystalline chalcopyrite absorber layers by Kelvin probe force microscopy, Nanotechnology 19 (14) (2008) 145705.

[28] M. Rusu, T. Glatzel, A. Neisser, C. Kaufmann, S. Sadewasser, M. C. Lux-Steiner, Formation of the physical vapor deposited $\mathrm{CdS} / \mathrm{Cu}(\mathrm{In}, \mathrm{Ga}) \mathrm{Se} 2$ interface in highly efficient thin film solar cells, Appl. Phys. Lett. 88 (14) (2006) 143510. 

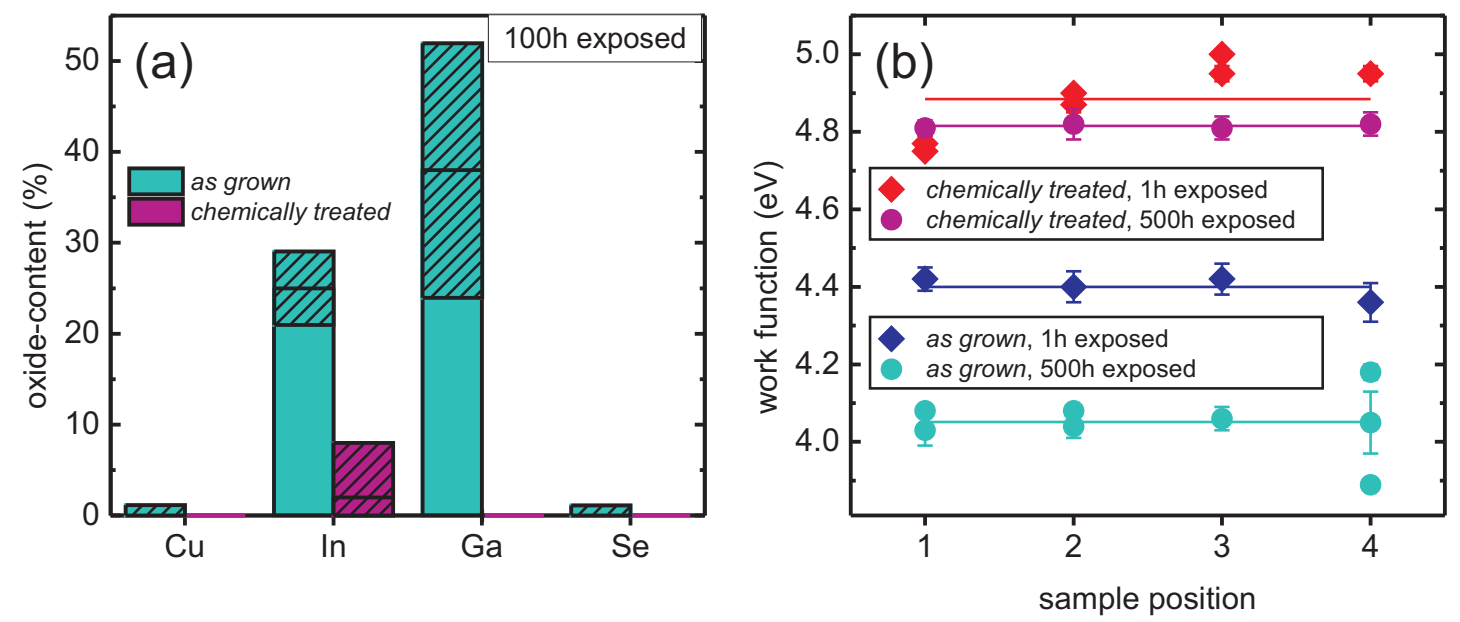

Figure 1: (a) XPS-determined surface oxide content of the individual elements of a CuIn $0.71 \mathrm{Ga}_{0.29} \mathrm{Se}_{2}$ thin film after $100 \mathrm{~h}$ exposure to ambient air as grown and chemically treated. The hatched areas indicate the error of the measurement. (b) Average work function of a CIGSe thin film after $1 \mathrm{~h} / 500 \mathrm{~h}$ exposure to ambient air as grown and chemically treated (measured on 4 macroscopically different sample positions). 

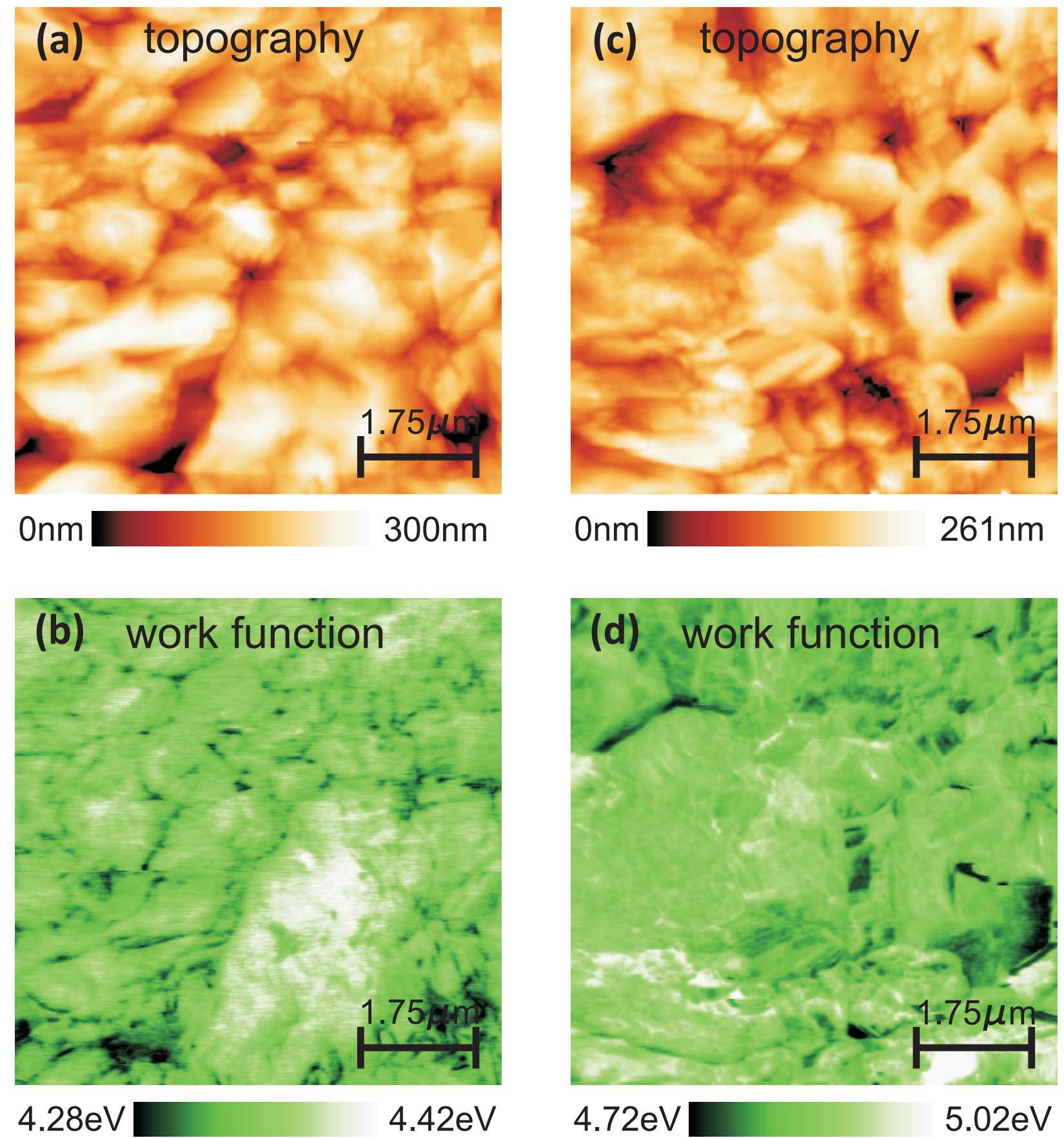

Figure 2: $7 \mu \mathrm{m} \times 7 \mu \mathrm{m}$ KPFM images of the CIGSe-0 thin film (a), (b) as grown and (c), (d) chemically treated 
CIGSe-0
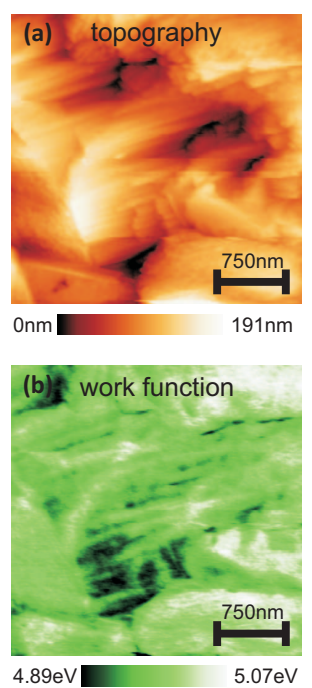

CIGSe-33
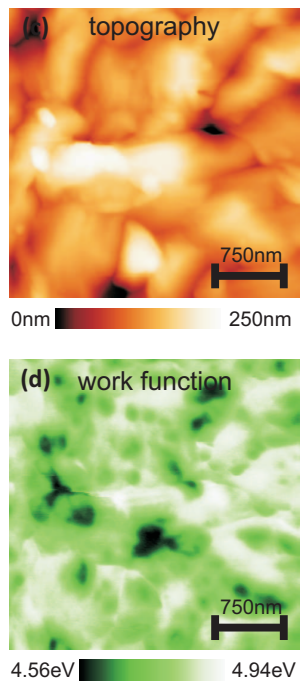

CIGSe-45
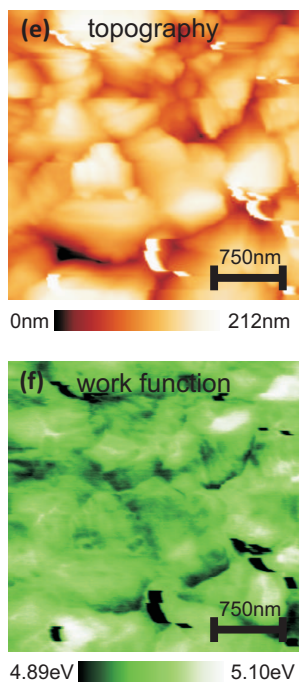

CIGSe-76
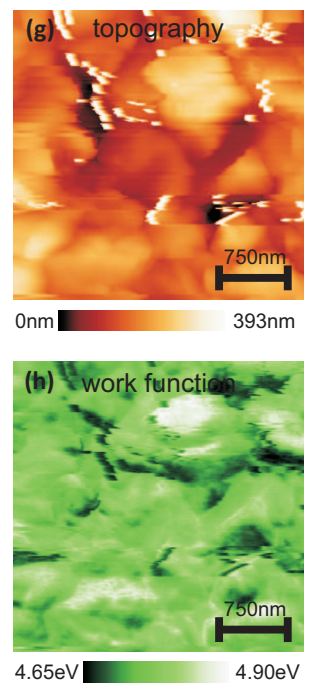

CIGSe-100
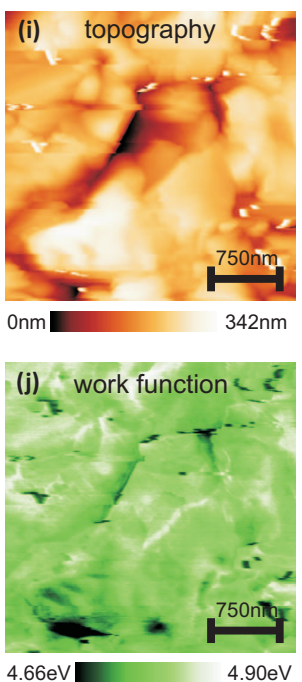

Figure 3: $3 \mu \mathrm{m} \times 3 \mu \mathrm{m}$ KPFM images of the CIGSe thin films with varying Ga-content. All films shown here are chemically treated. (a), (b) CIGSe-0; (c), (d) CIGSe-33; (e), (f) CIGSe-45; (g), (h) CIGSe-76; (i), (j) CIGSe-100

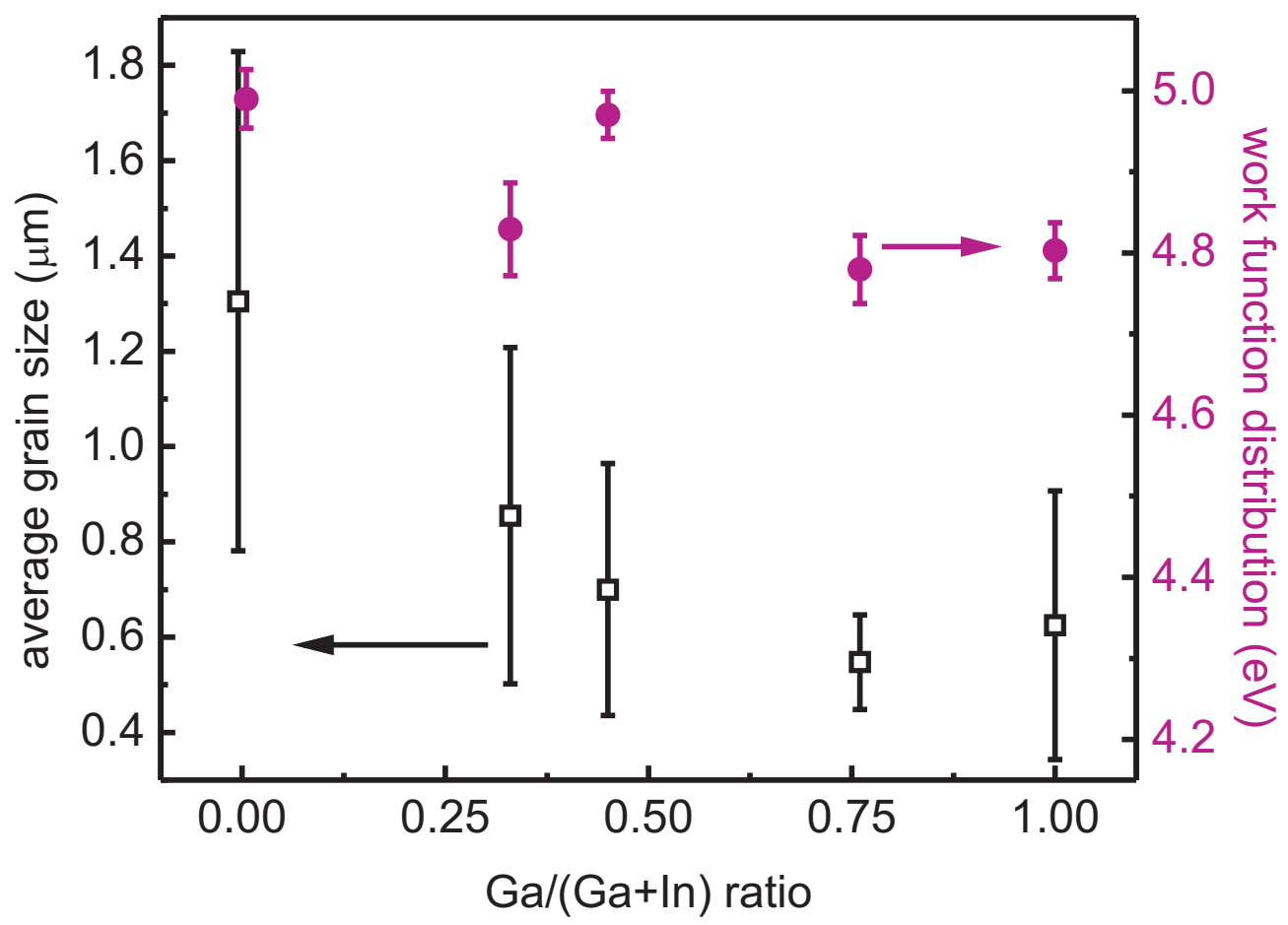

Figure 4: Average grain size and work function distribution in dependence of the Ga/(Ga+In) ratio. The error bars indicate the arithmetic mean of the grain size statistics and the FWHM of the work function distribution, respectively. 

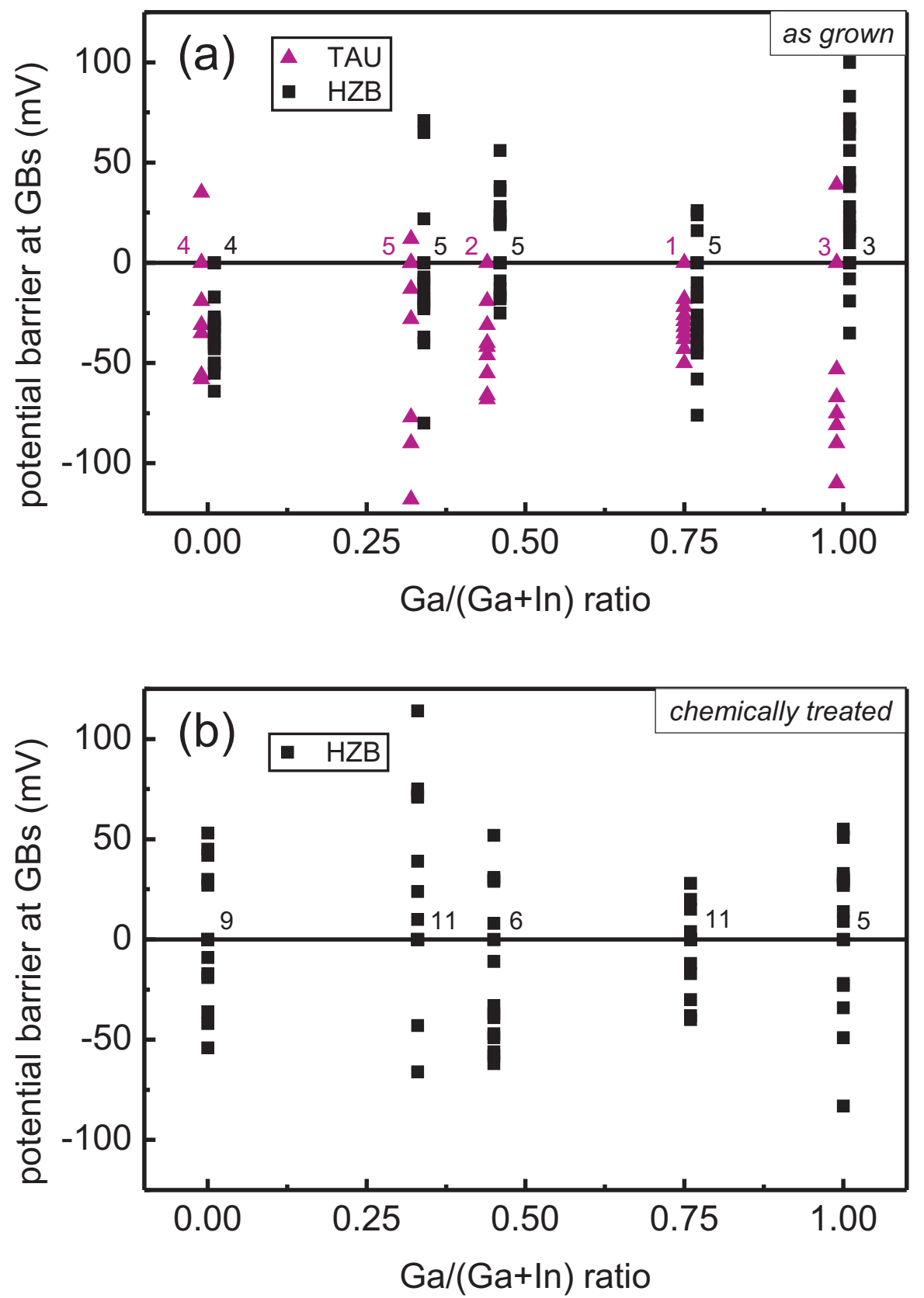

Figure 5: Electronic properties of GBs in dependence on the Ga/(Ga+In)-ratio for (a) as grown CIGSe thin films and (b) chemically treated CIGSe thin films. The number of electronically inactive GBs is indicated by black/purple numbers for each $\mathrm{Ga} /(\mathrm{Ga}+\mathrm{In})$-ratio. 